\title{
Probabilistic Approach to Determination of Dynamic Characteristics of Automatic Balancing Device
}

https://doi.org/10.1515/eng-2018-0033

Received July 16, 2018; accepted August 24, 2018

\section{Introduction}

Pumps are the most common type of machine used in industry of any country. Modern pumps can have sufficiently wide range of operating parameters, namely rotation frequency of some turbo pumps can reach tens of thousands of revolutions per minute, the pressure of pumped liquids up to hundreds of MPa. Different production requirements determine the variety of pump layouts and the design of their components. Automatic balancing device is one of such elements of high-pressure multi-stage pumps. It is used to unload the rotor from an unbalanced axial force acting on its impellers. Moreover, this element, in addition to its main unload function, performs an additional function of the end seal, reducing the fluid pressure at the pump outlet from the discharge pressure to the suction pressure.

The scheme of such typical balancing device is shown in Fig. 1. The force acting on the rotor is balanced by the hydrodynamic forces of the fluid pressure in the chamber 4 and in the throttle channel 3, which act on the rotating disk 5 (force $F$ ). The main elements of the such balancing device are successive cylindrical throttle 1 and face throttle 3. Face throttle resistance varies with the gap variation under possible axial displacements of the rotor which are caused by change in the axial force $T$ acting on the rotor. The latter ultimately provides a regulatory mechanism. At the same time, value of the face gap basically determines the leakage, which can be from 5 to $10 \%$ of the pump feed [1-3].

\footnotetext{
`Corresponding Author: Y. Tarasevych: Sumy State University, Ukraine, E-mail: tyulia@ukr.net

N. Sovenko, I. Savchenko: Sumy State University, Ukraine J. Skrzypacz: Wroclaw University of Science and Technologies, Poland
}

Ensuring reliable and economical operation of automatic balancing device is associated with a number of difficulties. It is rather complicated and sometimes impossible to increase the hydraulic resistance of a cylindrical throttle in practice that is why it is trying to increase the resistance of the face throttle usually. However, considering the dependence of the hydraulic resistance on the value of the face gap (from 20 to $200 \mu \mathrm{m}$ ), the necessity of its reducing is associated with the danger of scuffing during the operation of the pump. Quite high pressure drops on the face throttle of automatic balancing device (up to $10 \mathrm{MPa}$ ) lead to deformation of the support disk 5. Moreover, these deformations lead to the appearance of a diffusive gap, which in turn reduces the hydrostatic axial force on the face throttle. In addition, the deformations of the rotating disk 5 significantly affect not only the static, but also the dynamic behavior of the device as a whole. The large diffuser angle in the face throttle significantly reduces the stiffness characteristics of the liquid layer, and consequently, when axial vibrations of automatic balancing device disk rigidly connected to the rotor the additional hydrodynamic forces are taken place, caused by liquid layer in the gab, can lead to face oscillations and to the loss of dynamic stability. Therefore, when designing such devices, it is necessary to pay special attention to the calculation of hydrodynamic characteristics of both cylindrical and face throttle taking into account the above factors [1-6].

The field of application of automatic balancing device is rather narrow. Basically, these are multi-stage pumps for heat and nuclear power, as well as for the oil-producing and processing industries, which are not subject to modernization. Therefore, there are not so many papers devoted to theoretical research of this topic. Bases of static and dynamic characteristics calculation of automatic balancing devices were made in 1980's [3, 4, 7]. Later, on the basis of these works the specified results allowed to create the improved constructions of such devices $[1,8,9]$, but these constructions unfortunately did not get wide application in industry.

Flow rates through cylindrical and face throttles, as well as the forces and moments acting on the surface of 


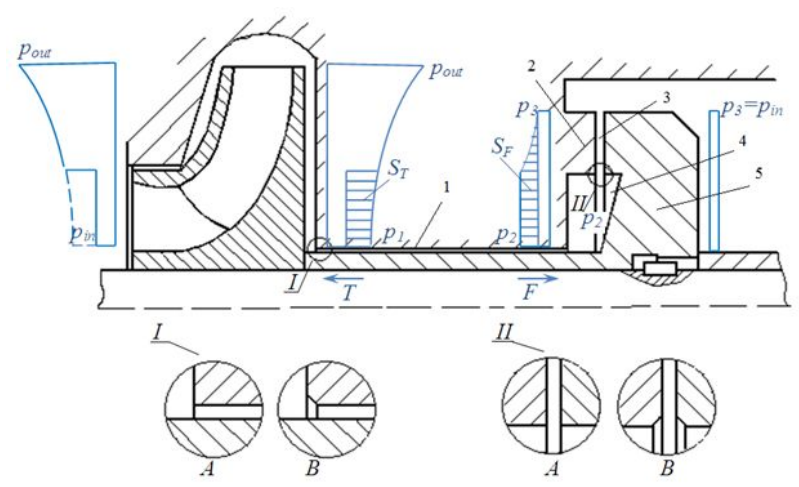

Figure 1: Scheme of typical automatic balancing device of centrifugal pump

the automatic balancing device from the pumped liquid, depend on the geometry of the gaps, the nature and velocities of movement of surfaces and fluid flow regimes [1]. Due to the manufacturing tolerances accepted in the pump construction, as well as the possible changes in the shape of the gaps during the operation, the values of the flow rates and forces calculated at the design stage may differ significantly from the actual ones. Well known and widely used analytical dependences to calculate the operational parameters of automatic balancing device are rather approximate and do not allow to take into account all the variety of factors affecting its operation. They are suitable for preliminary, engineering calculations and sometimes obtained results can be far from reality. Moreover, all calculated characteristics are usually obtained in the deterministic statement of the problem

Summary losses in the automatic balancing device can be more than $10 \%$ of pump efficiency. Modernization of the pump is usually associated with an improvement of working wheels and the flowing part geometry. This leads to increasing of pump efficiency by no more than $1-2 \%$. At the same time, as it is shown in [2,5], the influence of manufacturing tolerances and the changes in the shape of the gaps during the operation in cylindrical and face seals, which are random nature, can lead to deviations of the calculated characteristics to $10 \%$.

So, the main purpose of this work is to show the influence of random parameters (local hydraulic resistances, taper of face throttle) on dynamic characteristics of the automatic balancing device: natural frequency (amplitude) of the axial oscillations.

\section{Research Methodology}

The basic calculation of the automatic balancing device includes static and dynamic stages $[1,3,6]$. The purpose of the static calculation is to select the basic geometric parameters that determine the minimum flow rate for a given range of variation in the axial force $T$. The dependence of the face gap value on the axial force can be found from the condition of axial equilibrium of the unloading disc 5:

$$
T=F+F_{n},
$$

where $F=S_{2} p_{20}+S_{2} \Delta p_{b} \psi_{2}+F_{m}$ - resulting axial force of pressure, acting on disk 5 , which consists of the pressure force $p_{2}$ in chamber 4 and the pressure force in the face gap 3, $F_{n}=k_{d}\left(\Delta-h_{m 0}\right), k_{d}$ - the stiffness of the springs of the squeezing device, $\Delta$ - their preliminary compression, - value of the face gap. The pressure $p_{2}$ in the discharge chamber 4 depends on the face gap value $h_{m 0}$ and can be obtained from the equation of the balance of the flow of the liquid through the cylindrical $Q_{1}$ and face $Q_{2}$ throttles:

$$
Q_{1}=Q_{2}, g_{1} \sqrt{p_{1}-p_{2}}=g_{2} \sqrt{p_{2}-p_{3}},
$$

where $g_{1}$ and $g_{2}$ - conductivities of cylindrical and face throttles, which are determined by gap value of the corresponding throttle [3]. As it is shown in [2], due to the tolerances in the pumping design for the dimensions of the operating surfaces of the sealing pairs, the gap in the cylindrical throttle is a random variable, the range of changes of which can be $\pm(10 \ldots 30) \%$. Therefore, the conductivity of a cylindrical throttle has a random nature and is a random process if take into account the possible variation of the gap in time.

Static calculation is discussed in details in $[3,6]$.

The dynamic behavior of automatic balancing device with sufficient accuracy for practice is described by the differential equation of motion of the disk relative to the static equilibrium position for which the corresponding steadystate values of pressures, face gap and flow rate are determined under static calculation $[1,6]$ :

$$
m_{d} \ddot{z}_{d}+c_{d} \dot{z}_{d}+k_{d} z_{d}=S_{2} p_{20}+S_{2} \Delta p_{b} \psi_{2}+F_{m}-T+k_{d} \Delta
$$

where $m_{d}$ - rotor mass, $c_{d}$ - damping coefficient of axial rotor oscillations, $S_{2}$ - the surface area to which the pressure $p_{20}$ in the unloading chamber 4 is applied (value of pressure $p_{20}$ is obtained under static calculation).

Axial force of liquid pressure in face throttle can be determined by coefficients:

$$
F_{m}=F_{m 0}-A_{z} \ddot{z}_{d}-B_{z} \dot{\bar{z}}_{d}-C_{z} \bar{z}_{d}-B_{p} \dot{\psi}_{2}-C_{p} \psi_{2},
$$


$\bar{z}_{d}=z_{d} / h_{m 0}$ - dimensionless axial displacement of the disk, - dimensionless pressure in discharge chamber. The axial force of the fluid pressure in the face gap is caused by the pressure flow considering the local losses and the centrifugal flow (which determine the stiffness characteristics of the liquid layer), the shear flow caused by walls motion (damping characteristics) and the inertial flow (inertial characteristics), and is determined by the operating parameters (pressure and rotational frequency) and the geometry of the end throttle [6].

The equation of forced disk oscillations without considering the pressure changes in the balancing device chamber

$$
a_{11} \ddot{z}_{d}+b_{11} \dot{\bar{z}}_{d}+c_{11} \bar{z}_{d}=F_{0} e^{i \omega t},
$$

where $a_{11}=m_{d} h_{m 0}+A_{z}, b_{11}=c_{d} h_{m 0}+B_{z}, c_{11}=k_{d} h_{m 0}+$ $C_{z 0}+\chi_{\omega} \omega^{2}$.

$$
W(i \omega)=\frac{\bar{z}_{0} e^{i\left(\omega t+\phi_{1}\right)}}{F_{0} e^{i \omega t}}=A(\omega) e^{i \phi_{1}(\omega)} \rightarrow A(\omega)=
$$
$\frac{1}{\left(-a_{11} \omega^{2}+c_{11}\right)+i b_{11} \omega}$ - frequency system function; $A^{\prime}(\omega)=$ $\frac{\bar{z}_{d 0}}{\bar{z}_{d s}}=A(\omega) k=\frac{k_{d} h_{m 0}}{k_{d} h_{m 0}+C_{z 0}} \frac{1}{\sqrt{\left(1-v^{2}\right)^{2}+(2 \zeta v)^{2}}}$ - dynamical coefficient; where $\bar{z}_{d s}=F_{0} / k_{d} h_{m 0}$ - static axial displacement under amplitude value of force $F_{0}, v=\frac{\omega}{\omega_{0}}, \omega_{0}{ }^{2}=$ $\frac{k_{d} h_{m 0}+C_{z 0}}{m_{d} h_{m 0}+A_{z}+\chi_{\omega}} ; \chi_{\omega}$ - coefficient determined mainly by the value of the centrifugal flow and characterizing the change of liquid layer stiffness in the face throttle caused by rotational frequency.

The equation of oscillations should be supplemented with the equation of the balance of flow rates, which determines the dependence of the pressure in the chamber $\psi_{2}$ on the value of the face gap. The equation of the flow rates balance in contrast to the equations of statics takes into account the flow rate of displacement $Q_{v}$ and compression $Q_{p}$ in the discharge chamber

$$
Q_{1}=Q_{2}+Q_{v}+Q_{p}
$$

where $Q_{v}=S_{2} z_{d}$ - changing the chamber volume and $Q_{p}=$ $\frac{V}{E} \dot{p}_{2}$ - change in the volume of fluid in the chamber from the compression of the fluid under axial displacements of the rotor. In addition, the flow rate through the face throttle $Q_{2}$ is determined not only by the pressure flow, but also depends on the oscillations of the face gap [3].

The value of flow rate both through the cylindrical and through the face throttle depends on the value of the resistances, as well as the shape of the gap. The geometry of the gap, which can be non-flat initially due to inaccurate manufacturing and possible distortions during installation, may also change during operation. In addition, to take into account the effect of resistances, appropriate coefficients are used, the values of which are determined empirically [4]. However, it should be noted that their actual values may differ significantly from the calculated ones because of the lack of requirements for the shape of the inlet cross-sections of the cylindrical and face throttles. Different shapes of the input sections are possible in different assemblies and variants of installation of the discharge disc of automatic balancing device (see Figure 1). In this case, the sealing surfaces can change its shape due to wear and force deformation during operation of the pump and this means that the coefficients of local losses are random variables by their nature.

So, corresponding loss factors and also the parameter characterizing the shape of the cross-section of face throttle will be considered as random values under calculating the dynamic characteristics of the automatic balancing device. At the moment there is no information on what distribution laws these random variables can have. So, on the basis of the central limit theorem of probability theory, as the first approximation in the solution of the probabilistic problem the normal distribution laws will be taken for the indicated random variables. And just as it was done in work [5] empirical values of the considered coefficients will be taken as mean values and the corresponding standard deviations will be given by the percentage deviation of the values of the considered random variable from its mean value determined by the definition physics problems [4]: $\left\langle\xi_{\text {im }}\right\rangle=1.5,\left\langle\xi_{\text {om }}\right\rangle=0.2\left\langle\xi_{\text {im }}\right\rangle$. Here $\langle X\rangle=\int_{-\infty}^{\infty} f(x) x d x$ - the mean value operator applied to the corresponding random variable.

To determine the effect of a random variation of these parameters on the inertia, damping and stiffness coefficients caused by action of hydrodynamic forces on the surface of automatic balancing device from the flow the joint probability density $f_{1}\left(\zeta_{i m}, \zeta_{o m}, \zeta_{2 m}, \beta\right)$ take into considering. This joint probability density, according to the stochastic independence of the random variables, can be represented in the form

$$
f_{1}\left(\zeta_{i m}, \zeta_{o m}, \zeta_{2 m}, \beta\right)=f_{2}\left(\zeta_{i m}\right) f_{3}\left(\zeta_{o m}\right) f_{4}\left(\zeta_{2 m}\right) f_{5}(\beta)
$$

where $f_{2}\left(\zeta_{i m}\right), f_{3}\left(\zeta_{o m}\right), f_{4}\left(\zeta_{2 m}\right)$ - the normal probability density of the coefficients of local hydraulic losses at the inlet $\zeta_{i m}$, at the outlet $\zeta_{o m}$ and along the length of face throttle $\zeta_{2 m}, f_{5}(\beta)$ - the probability density of the taper angle of the face throttle.

For practical use, it is sufficient to know the first two moment characteristics of the natural frequency of the axial oscillations of the automatic balancing device: mean value and the standard deviation. The problem of finding the probability density of the natural frequency is not presented in this paper. 
Table 1

\begin{tabular}{ccccc}
\hline \multirow{2}{*}{ Tolerance } & \multicolumn{4}{c}{ Possible range of variable } \\
\cline { 2 - 5 } & $\zeta_{\text {im }}$ & $\zeta_{\text {om }}$ & $\beta$ & $\omega_{0}$ * $10^{-3}$ \\
\hline $5 \%$ & $(1.275 ; 1.725)$ & $(0.255 ; 0.345)$ & $(0.095 ; 0.128)$ & $(2.8 ; 3.15)$ \\
$10 \%$ & $(1.05 ; 1.95)$ & $(0.21 ; 0.39)$ & $(0.078 ; 0.145)$ & $(2.62 ; 3.32)$ \\
$15 \%$ & $(0.825 ; 2.175)$ & $(0.165 ; 0.435)$ & $(0.061 ; 0.161)$ & $(2.42 ; 3.5)$ \\
$20 \%$ & - & $(0.12 ; 0.48)$ & $(0.044 ; 0.178)$ & $\left.(2.7 ; 3.2)\right|_{\zeta_{\text {im }}-5 \%}$ \\
$30 \%$ & - & - & $(0.011 ; 0.211)$ & $\left.(2.59 ; 3.38)\right|_{\zeta_{\text {im }}-10 \% \zeta_{\text {om }}-10 \%}$
\end{tabular}

From (5) natural frequency of axial disk oscillations

$$
=\sqrt{\frac{\omega_{0}=\sqrt{\frac{c_{11}}{a_{11}}}}{m_{d} h_{m 0}+A_{z}\left(\zeta_{i m}, \zeta_{o m}, \zeta_{2 m}, \beta\right)+\chi_{\omega}\left(\zeta_{i m}, \zeta_{o m}, \zeta_{2 m}, \beta\right)}}
$$

Mean value of natural frequency can be obtained based on the formula

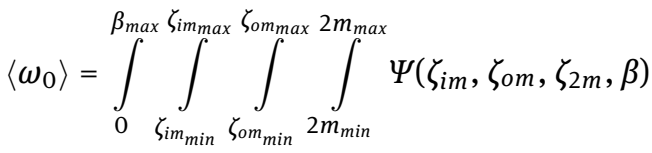

$$
\begin{aligned}
& f_{1}\left(\zeta_{i m}, \zeta_{o m}, \zeta_{2 m}, \beta\right) d \zeta_{2 m} d \zeta_{o m} d \zeta_{i m} d \beta
\end{aligned}
$$

The standard deviation:

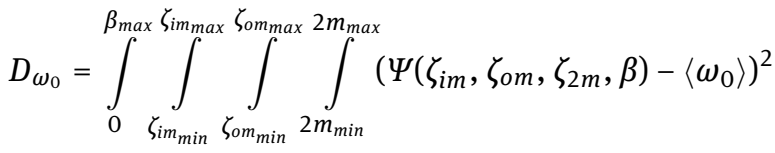

$$
\begin{aligned}
& f_{1}\left(\zeta_{\text {im }}, \zeta_{o m}, \zeta_{2 m}, \beta\right) d \zeta_{2 m} d \zeta_{o m} d \zeta_{i m} d \beta
\end{aligned}
$$

According to the studies, the range of possible values of natural frequency increases with increasing the standard deviations of random values $\zeta_{i m}, \zeta_{\text {om }}, \zeta_{2 m}, \beta$ (Table 1 ). Numerical calculation was made for the following values of basic parameters $r_{m}=0.089 \mathrm{~m}, p_{1}=35 \cdot 10^{6} \mathrm{~Pa}, h_{m o}=$ $88 \mu \mathrm{m}, m_{d}=10 \mathrm{~kg}, k_{d}=10^{3} \mathrm{~kg} \cdot \mathrm{s}^{-1}, c_{d}=5 \cdot 10^{6} \mathrm{~kg} \cdot \mathrm{s}^{-2}$. According to calculation the deterministic value of natural frequency is $\omega_{0}=2.99 \cdot 10^{3} \mathrm{c}^{-1}$.

The dependences of dimensionless face gap $u=h_{m 0}+$ $z_{d}$ on external force can be determined from equation (5), (6) system in a way

$$
\begin{aligned}
& a_{11} \ddot{\bar{z}}_{d}+b_{11} \dot{\bar{z}}_{d}+c_{11} \bar{z}_{d}+b_{12} \dot{\Psi}_{2}+c_{12} \Psi_{2}=F_{a} \\
& a_{21} \ddot{\bar{z}}_{d}+b_{21} \dot{\bar{z}}_{d}+c_{21} \bar{z}_{d}+b_{22} \dot{\Psi}_{2}+c_{22} \Psi_{2}=0,
\end{aligned}
$$

From (9) dynamic coeffisient for axial oscillations:

$$
A_{d}(\omega)=\bar{z}_{d 0} / \bar{z}_{d s}=\frac{\left|W_{d}(i \omega)\right|}{A_{s}}
$$

and

$$
\varphi_{d}(\omega)=\arctan \left(\frac{\operatorname{Re}\left[W_{d}(i \omega)\right]}{\operatorname{Im}\left[W_{d}(i \omega)\right]}\right),
$$

or

$$
\begin{aligned}
& A_{d}(\omega)=\bar{z}_{d 0} / \bar{z}_{d s}=\frac{1}{A_{s}} \sqrt{\frac{U_{22}^{2}+V_{22}^{2}}{U^{2}+V^{2}}} \\
& \varphi_{d}(\omega)=-\arctan \left(\frac{U_{22} V+V_{22} U}{U_{22} U+V_{22} V}\right)
\end{aligned}
$$

where

$$
\begin{aligned}
& U_{11}=-\left(a_{11}-\chi_{\omega}\right) \omega^{2}+c_{110}, \\
& V_{11}=b_{11} \omega \text {, } \\
& U_{12}=c_{12} \text {, } \\
& V_{12}=b_{12} \omega \text {, } \\
& U_{22}=c_{22} \text {, } \\
& U_{12}=-a_{21} \omega^{2}+c_{21} \text {, } \\
& V_{21}=b_{21} \omega \text {, } \\
& V_{33}=b_{22} \omega \text {, } \\
& \begin{array}{c}
U=\left(-a_{11} c_{22}-b_{11} b_{22}+a_{21} c_{12}+b_{21} b_{12}\right) \omega^{2} \\
+\left(c_{11} c_{22}-c_{21} c_{12}\right),
\end{array} \\
& V=\left(-a_{11} b_{22}+a_{21} b_{12}\right) \omega^{2}+\left(b_{11} c_{22}-b_{21} c_{12}\right) \omega \\
& +\left(b_{22} c_{11}-b_{12} c_{21}\right) \text {; } \\
& b_{12}=B_{p} \text {, } \\
& c_{12}=C_{p}-S_{2} p_{20}, \\
& c_{21}=Q_{m o} v_{z} \text {, } \\
& a_{21}=-Q_{m 0} \frac{p r_{m} b}{4 \Delta p_{20}} \frac{\zeta_{m 0}}{\zeta_{m 0}-\zeta_{2} n / 2} v_{g_{z}} \text {, } \\
& b_{21}=-2 \pi\left[v_{c z}-(1-\Lambda)^{2}\right] \frac{r_{m} h_{m 0}}{2}+S_{2} h_{m 0} \text {, } \\
& c_{22}=Q_{m 0} \frac{p_{20}}{2}\left(\frac{1}{p_{1}-p_{20}}+\frac{1}{\Delta p_{20}}\right) \text {, } \\
& b_{22}=-Q_{m 0} \frac{p r_{m} b}{4 \Delta p_{20}} \frac{\zeta_{m 0}}{\zeta_{m 0}-\zeta_{2} n / 2} \frac{p_{20}}{k_{r 0} \mu}+\frac{V_{2} p_{20}}{E} \text {. }
\end{aligned}
$$




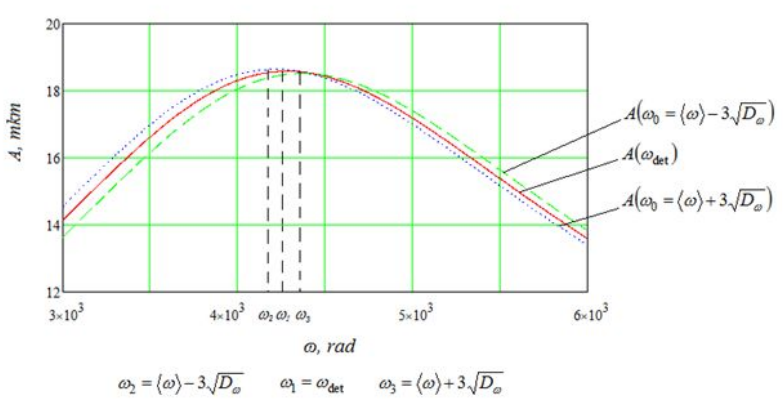

Figure 2: Amplitude-frequency characteristics of automatic balancing device with taking into account random changes of basic parameters of face throttle

On Fig. 2 presented typical amplitude-frequency characteristics of automatic balancing device with taking into account random changes in the stiffness and inertia coefficients.

If the changes result in a decrease in the natural frequency, this may lead to the operation of the device with increased values of the oscillation amplitudes in comparison with the calculated values, which in turn can lead to scuffing and failure of the device.

\section{Conclusions}

The results obtained in the work confirmed the necessity of considering the stochastic nature of the basic parameters of the face throttle of the automatic balancing device in the calculation of its dynamic characteristics. So, the possible deviation of natural frequency value from deterministic one makes from 7 (under $5 \%$ deviation interval of random values $\left.\zeta_{i m}, \zeta_{i m}, \zeta_{2 m}, \beta\right)$ to $17 \%$ (under 15\%). Decreasing of natural frequency value results in reduction of operating range of device with the small values of amplitudes of force vibrations.

The calculations given in this paper can be regarded as the first approximation to the solution of this problem. The result showed quantitative influence of the random changes of the hydraulic resistances and taper of face gap on the values of the natural frequency of balancing disk. It is important to note the fact that the deviation of the taper value in a smaller side from the calculated value can lead to negative consequences: displacement of natural frequency value to lower range with increasing of amplitudes values can provide to scuffing and total failure of whole device. And as it shown in other works of authors - to decreasing in the volumetric efficiency of the pump. In subsequent studies, one should consider the effect of a random change in the parameters of the cylindrical gap of the balancing device to its characteristics. The hydrodynamics of a cylindrical gap largely determines the pressure in the discharge chamber, which in turn is determined by the face gap value.

\section{References}

[1] Korczak A., Badania ukladow rownowazacych napor osiowy w wielostopniowych pompach odsrodkowych, 2005, Gliwice: Wyd. Politechniki Slaskiej.

[2] Tarasevich Y., Savchenko I., Sovenko N., Savchenko A., Research of influence of random change of annular seal parameters on efficiency of centrifugal pump, Eastern-European Journal of Enterprise Technologies, 2016, 6/7, 84, 37-42.

[3] Martsinkovskii V., Vorona P., Nasosy atomnyh elektrostancii, 1987, Moscow: Energoatomizdat.

[4] Jedral W., Turbulentny przeplyw cieczy w hydraulicznie gladkich szczelinach poprecznych. Archiwum budomy maszyn, 1981, XXVIII, 1.

[5] Tarasevich Y., Sovenko N., Savchenko I., Influence of the stochastic nature parameters of throttle channels on characteristic of automatic balancing device of the centrifugal pump. Lecture Notes in Mechanical Engineering (Springer), 2019.

[6] Zuyeva N., Calculation method of the improved design of automatic balancing device with hydraulically unloaded resiliently mounted ring. Procedia Engineering, 2012, 39, 387-394.

[7] Chegurko L., Razgruzochnye ustrojstva pitatelnyh nasosov teplovyh elektrostancij, 1978, Moskva: Energija.

[8] Korczak A., Zarzycki M., Perchał S., Peczkis G., Pompa odśrodkowa wielostopniowa. Zgłoszenie patentowe P-354697 Z 24.06.02.

[9] Korczak A. Marcinkowski W.A. Peczkis G., Tarcza odciążająca siłę osiową w sprężarce wirnikowej. Zgłoszenie patentowe P365432. 20.02.2004. 